\title{
(-)-ISOPINOCAMPHEOL SUBSTITUTED MESOGENS: AN INVESTIGATION OF THE EFFECT OF BULKY TERMINAL GROUPS IN CHIRAL SMECTIC LIQUID CRYSTALS
}

\author{
C. Yates ${ }^{1}$, J. P. F. Lagerwall ${ }^{2}$, M. Nobili ${ }^{3}$, S. Rauch ${ }^{1}$, G. Heppke ${ }^{1}$ \\ ${ }^{1}$ TUB, Stranski-Laboratorium, Sekr. ER11, Straße des 17 Juni 135, 10623 Berlin, \\ Germany \\ ${ }^{2}$ Universität Stuttgart, Institut für Physikalische Chemie, Pfaffenwaldring 55, 70569 \\ Stuttgart, Germany \\ ${ }^{3}$ Groupe de Dynamique des Phases Condensées Université de Montpellier II, Place E. \\ Bataillon, 34000 Montpellier, France
}

\begin{abstract}
A ferroelectric liquid crystal with an end-substituted (-)-isopinocampheol (IPC) group has been studied in isolation and in mixtures with the antiferroelectric compound EHPOCBC. The samples were studied with respect to electroclinic coefficients, tilt angles, X-ray layer spacing, dielectric permittivity, spontaneous polarisation and relative birefringence under an applied field. In the pure isopinocampheol substituted compound, the $\mathrm{SmA}^{*}-\mathrm{SmC}^{*}$ phase transition appears strongly first order and is associated with a significant increase in effective birefringence. The smectic layer spacing decreases below the transition approximately with the optical tilt. On increased EHPOCBC concentration the change in birefringence across the transition diminishes, until the point at which the mixture adopts an anticlinic $\mathrm{SmC}_{\mathrm{A}}{ }^{*}$ phase, and the expected decrease in birefringence is observed. There is very little change in birefringence with field for the IPC compound, whilst EHPOCBC shows a marked increase with increasing field, for a few degrees above the phase transition.

The results are analyzed with respect to two models. In one it is proposed that the bulky IPC group disorders the SmA* phase by introducing undulations in the smectic layer. These undulations may disappear in the $\mathrm{SmC}^{*}$ phase due to an antiparallel arrangement of neighbouring molecules. In the other model the bent shape of the mesogen as a whole plays a crucial role in changing the optical properties at the $\mathrm{SmA}^{*}-\mathrm{SmC}^{*}$ transition, due to the biased rotation around the molecular axis of inertia.
\end{abstract}

Keywords: de Vries, smectic A*, birefringence, bulky substituents

\section{INTRODUCTION}

In recent years there has been a growing interest in ferro- and antiferroelectric liquid crsytal (FLC and AFLC, respectively) materials that show negligible layer shrinkage on passing through the $\mathrm{SmA}^{*}-\mathrm{SmC}^{*}\left(\mathrm{SmC}_{\mathrm{A}}{ }^{*}\right)$ phase transition [1-6]. The majority of commercial and academic materials exhibit a considerable layer contraction, with the layer thickness in $\mathrm{SmC}^{*}$ often being reduced approximately by a factor $\cos (\theta)$, where $\theta$ is the optical director tilt angle. It is generally agreed that the mismatch between the layer spacing at the surfaces of a planar aligned cell and the bulk layer thickness resulting from this layer contraction causes the formation of vertical chevrons within the cell, and the concomitant zig-zag textural defects [7]. Hence, it is expected that a material with zero layer shrinkage would eliminate the source of these defects, a major problem in the commercialisation of FLC displays. 
In this connection, the models of SmA* phases promoted by de Vries [8] are especially attractive, since they assume a non-zero molecule tilt in the $\mathrm{SmA}^{*}$ phase, the uniaxial symmetry around the layer normal arising from the absence of long range correlation of the tilting direction, i.e. in the azimuthal angle. The progression to the $\mathrm{SmC}^{*}$ phase can then correspond to a biasing of a preferred azimuthal orientation, a transition type that de Vries gave the name asymmetric diffuse cone model transition (in the following referred to as ADC model). It is clear that materials exhibiting such a tilting transition could be expected to show small or negligible layer shrinkage in the SmC* phase. In addition, this type of transition leads to some rather characteristic optical properties. The 'fingerprint' of an ADC model smectic can thus be summarised as follows, i) negligible smectic layer shrinkage on passing through the $\mathrm{SmA}^{*}-\mathrm{SmC}^{*}$ phase transition, ii) a large electroclinic coefficient and field-induced polarisation, iii) a significant increase in birefringence with applied field in the SmA* phase, iv) a significant increase in birefringence on passing from $\mathrm{SmA}^{*}$ to a helix-free $\mathrm{SmC}^{*}$ phase (helix expelled by surface or field action), possibly proportional to the tilt of the optic axis.

Unfortunately, materials which show the property of negligible layer shrinkage, usually identified by X-ray layer spacing measurements, are few and the molecular design constraints are not yet clear. One (empirically based) suggestion in this direction is that molecules which are asymmetric along the long axis of the molecule, with one end group considerably bulkier than the other, might promote an ADC model SmA*-SmC* transition [1]. In this paper, we have therefore chosen to study an isopinocampheol substituted compound (IPC 515) exhibiting a SmA*-SmC* phase sequence, in comparison to the well known antiferroelectric compound EHPOCBC, exhibiting a direct $\mathrm{SmA}^{*}-\mathrm{SmC}_{\mathrm{A}}$ * transition. The chemical structures and phase sequences are shown in Fig.1. The isopinocampheol group is both bulky and rigid so IPC515 could be a candidate for an ADC model material. However, the rather long achiral alkyl chain on one side, and the absence of a chain on the other, gives the molecule on the whole a fairly distinct bent shape, at least assuming that the alkyl chain on the average adopts its all trans conformation, which makes the analysis of the behaviour of this compound slightly complicated. The isopinocampheol group might also have the ability to disrupt interlayer correlations, as compared to ordinary mesogenic molecules with a flexible chain on each side. EHPOCBC was chosen as the comparative material due to the fact that it possesses an identical mesogenic core to IPC 515 and hence differences in birefringence between the compounds can be attributed to the order of the cores (on a local as well as a mesoscopic scale).

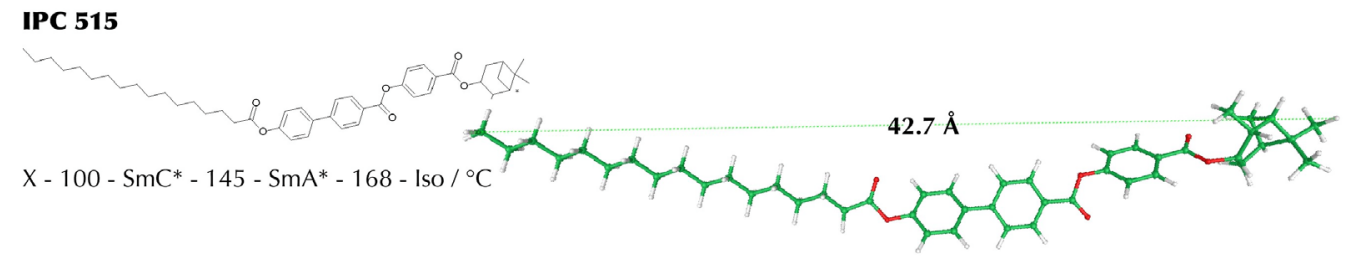

(S)-EHPOCBC
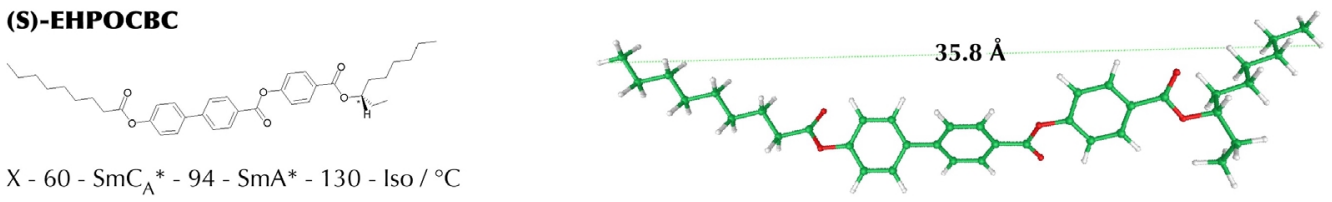

Figure 1. Chemical constitutions and phase sequences of the studied compounds. 
In order to achieve a high packing efficiency when the molecules are much bulkier at one end than at the other, we can imagine two basic packing schemes. These are illustrated, in an extremely schematic way, in Figure 2. The molecule structure used as a 'building block' in this figure is the IPC515 molecule in the conformation resulting from a MOPAC energy minimization. On the one hand one could imagine that the molecules prefer organizing core to core and tail to tail. The bulky end group on one side would then lead to a splayed 'flower bouquet' organization as illustrated to the left. This organization corresponds first of all to a local splay in the director field, second it would seem to violate the sign invariance of the director (n). In order to compensate the splay and retain the $\mathbf{n}=-\mathbf{n}$ condition an 'up' bouquet must therefore be followed by a 'down' bouquet, in three dimensions resulting in a structure something like an egg box. Another possibility is that the molecules want to compensate the asymmetry on an as small a scale as possible, i.e. neighboring molecules pack antiparallel to one another, as illustrated in Figure $2 \mathrm{~b}$. It should be pointed out that this discussion of packing efficiency is quite fictitious as it completely neglects the thermal motion. The pictures are useful for simplifying the discussion, but should be understood as applying on a very local scale (groups of $\sim 10$ molecules) only and also then only partially.
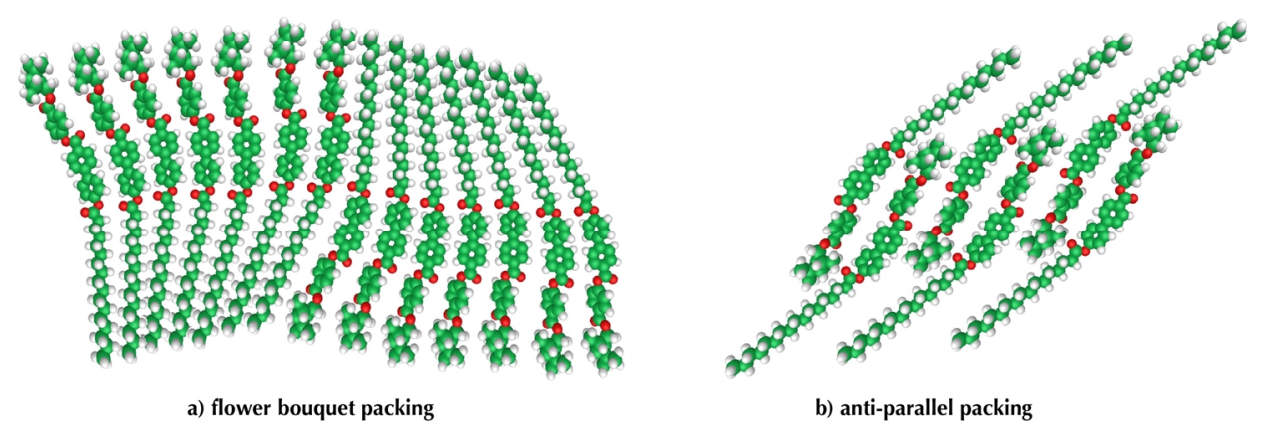

Figure 2. Possible molecular close-packing schemes, illustrated extremely schematically.

\section{EXPERIMENTAL}

All optical measurements were taken using standard EHC cells, of various thicknesses, with a rubbed polyimide alignment layer. Optical tilt angles were measured using a technique first proposed by Bahr and Heppke where the optical transmission $(T)$ of both states during saturated square wave $(10 \mathrm{~Hz})$ switching was measured for several consecutive sample orientations $\varphi$. By fitting two $\sin ^{2}$ functions to the two resulting $T(\varphi)$ data sets the tilt angle can be extracted [9]. The electroclinic coefficients in the $\mathrm{SmA}^{*}$ phase were extracted subsequently from linear fits of the tilt versus field. The spontaneous polarisation was measured by the triangular wave polarisation reversal method [10]. For the X-ray layer spacing measurements the crystalline sample was ground to a fine powder and then filled into Lindmann capillary tubes of $1 \mathrm{~mm}$ diameter. During measurements the tubes were kept in a Mettler oven with $0.1{ }^{\circ} \mathrm{C}$ temperature resolution. Each sample was heated into the isotropic phase and then the temperature was decreased at $3^{\circ} \mathrm{C} / \mathrm{min}$. At each measurement temperature the sample was allowed to equilibrate for ten minutes before the measurement was performed. The relative birefringence was measured at $656 \mathrm{~nm}$ by taking the ratio of transmitted light for a sample between crossed and parallel polarisers [11]. 


\section{RESULTS AND DISCUSSION}

Pure IPC 515.

The measured smectic layer spacing, optical tilt and the tilt angle calculated from a power law fit to the X-ray data are shown, as a function of temperature, in Figure 3. The layer spacing in the $\mathrm{SmA}^{*}$ phase is $\sim 42 \AA$, which is close to the all trans molecular length of $\sim 43 \AA$. This might seem to indicate that the diffuse cone model is not applicable, but it could also mean that the molecules aggregate in antiparallel pairs, such that the effective length of the 'rods' building up the layers is considerably longer than one molecule, $c f$. Figure $2 \mathrm{~b}$. In order to conclude which is the case a measurement of the orientational order parameter, for instance by means of 2D imaging of $\mathrm{x}$-ray scattering from aligned samples, would have to be carried out.

It is immediately clear that there is a significant shrinkage of the smectic layers below the $\mathrm{SmA}^{*}-\mathrm{SmC}^{*}$ transition. Furthermore, there is actually a quite close correspondence between the optical tilt and the x-ray tilt. Hence, the transition is obviously related to an actual increased molecular tilt, i.e. the transition is not an ADC model transition. Both tilt angles have a similar small critical exponent, $\left(\gamma_{\text {optical }}=0.24\right.$, $\gamma_{X \text {-ray }}=0.30$ in a fit of the power law $\left.\theta=C\left(T_{C}-T\right)^{\gamma}\right)$, indicating the first order nature of the transition. The first order nature is also emphasised by the dielectric spectroscopy results, where there was no trace of the soft mode in the $\mathrm{SmA}^{*}$ phase as would be expected for a second order transition.
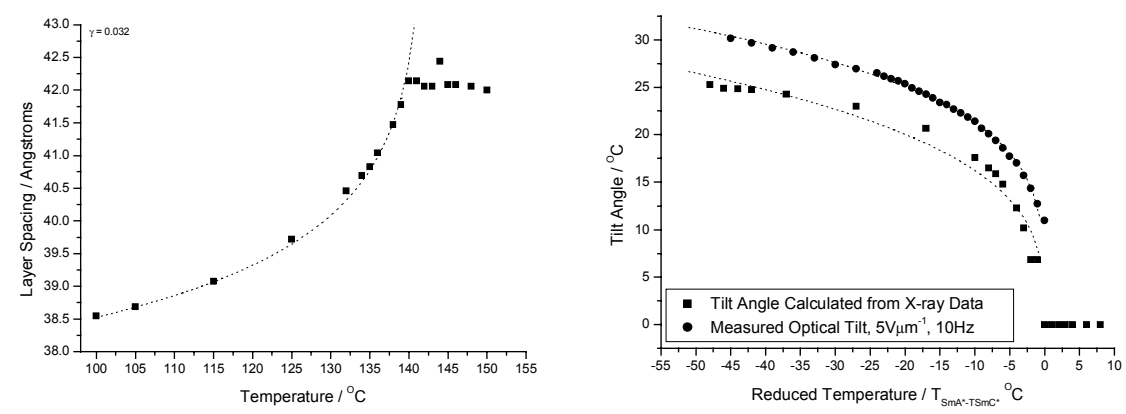

Figure 3. X-ray layer spacing and optical tilt compared to calculated tilt from layer shrinkage, for IPC 515.

When the compound was filled into cells for electrooptic measurements we noticed that the helix was easily expelled even in cells considerably thicker than the helical pitch length. In homeotropic alignment the $\mathrm{SmC}^{*}$ phase gave visible selective reflection, signifying a pitch length of less than $0.5 \mu \mathrm{m}$. But in a $6 \mu \mathrm{m}$ thick planaraligning cell the helix was partially expelled in the virgin $\mathrm{SmC}^{*}$ state. After switching, the sample generally relaxed to a twisted state, as could easily be seen by the absence of any orientation giving extinction between crossed polarizers. The ease in disrupting the ordinary smectic helix along the layer normal by surface action is an indication that the interlayer correlations in this compound are indeed weak, a characteristic which may be attributed to the isopinocampheol terminal group, as discussed above.

The phase diagram for the mixture of IPC 515 and EHPOCBC is shown in Figure 4. The transition from FLC behavior on the IPC515-rich side to AFLC behavior on the 
EHPOCBC-dominated side was mediated via a very narrow region where the chiral smectic $\mathrm{C}$ subphases were induced. So far, this conclusion is based solely on the homeotropic texture observation in a contact sample and, although there is no question that at least one of the long-pitch $\mathrm{SmC}_{\beta}$ * or $\mathrm{SmC}_{\gamma}$ * subphases (easily recognized by their characteristic schlieren texture) was formed, we cannot at this stage say exactly which subphases were induced or what their temperature ranges are. This is the topic of an ongoing study.

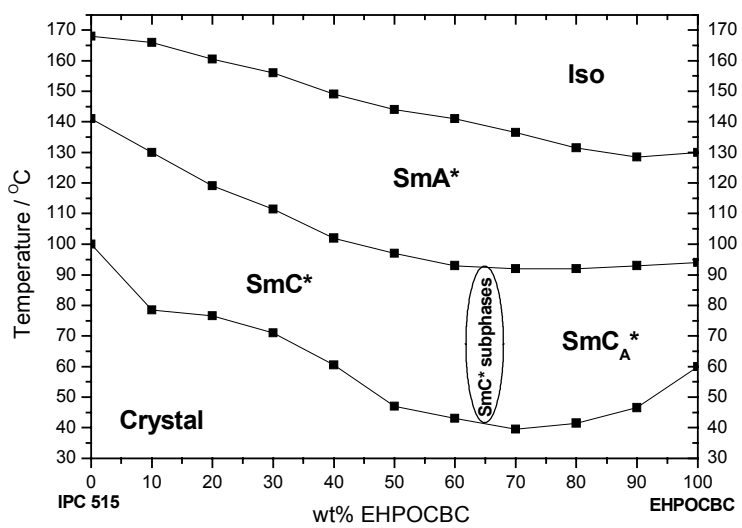

Figure 4. Phase diagram for mixtures of IPC 515 and EHPOCBC.

The variation of the electroclinic coefficient with temperature for the mixtures of IPC 515 and EHPOCBC, are shown in Figure 5, together with the measured values of polarisation. It is worth noting that all the mixtures possessing a $\mathrm{SmC}_{\mathrm{A}}{ }^{*}$ phase have larger coefficients than the SmC* materials. Starting from pure IPC 515, the electroclinic coefficient decreases on increasing the amount of EHPOCBC, passing through a minimum at about $30 \mathrm{wt} \%$ and then increasing to pure EHPOCBC. This is due to $(s)$-EHPOCBC and $(r, r, r, s)$ - IPC 515 having opposite signs of spontaneous polarisation. The connection between electroclinic coefficient and induced polarisation is clear, with the values for $\mathrm{SmC}_{\mathrm{A}}{ }^{*}$ samples exceeding those for the $\mathrm{SmC}^{*}$ samples.

One should point out that the strong first-order nature of the $\mathrm{SmA}^{*}$-SmC* transition in IPC 515 makes it possible to field-induce the transition, as was clearly verified during texture observations while switching the sample at temperatures slightly above the zero-field transition temperature. Hence, it is not obvious if the field-induced tilt measured close to the transition is really an electroclinic SmA*-tilt or if it arises as an average of regions still in $\mathrm{SmA}^{*}$ and other regions where the $\mathrm{SmC}^{*}$ phase has been induced. This may explain the discrepancy between electrooptic and dielectric measurements, the soft mode being the dielectric spectroscopy equivalent of the electroclinic effect. As there was essentially no soft mode in the dielectric spectra from IPC515 the rather large apparent electroclinic coefficient measured close to the phase transition is quite surprising. 

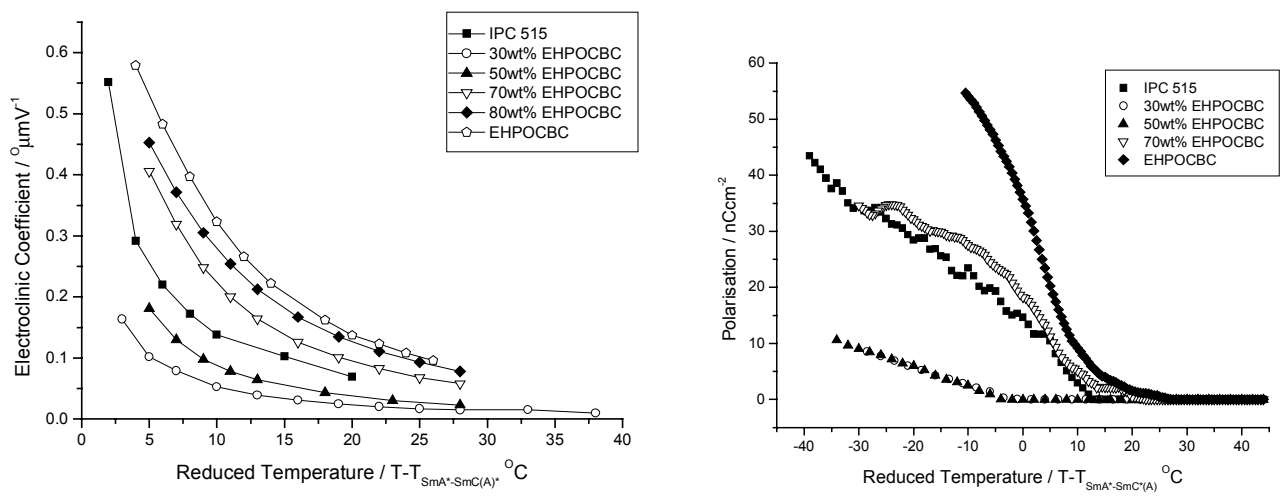

Figure 5. Electroclinic coefficients and measured polarisation for mixtures of IPC 515 and $\mathrm{EHPOCBC}$.

The relative changes in birefringence on cooling for mixtures of IPC 515 and EHPOCBC are shown in Figure 6. In this figure the measured birefringence has been scaled such that at the phase transition the birefringence is set equal to zero. This allows a quantitative comparison of the relative changes as the samples are cooled. The most striking aspects of this plot are the SmC* increase in birefringence for both pure IPC 515 and $30 \mathrm{wt} \%$ EHPOCBC in IPC 515 and the corresponding decrease for the $70 \%$ and $100 \%$ EHPOCBC samples. All samples were helix-free due to surface stabilization in the $2 \mu \mathrm{m}$ thick samples. The decrease in birefringence of EHPOCBC is explained by the appearance of an anticlinic arrangement of the optical director tilt angle, where the observed decrease in birefringence corresponds to a director tilt in the region $14-17^{\mathrm{O}}$.

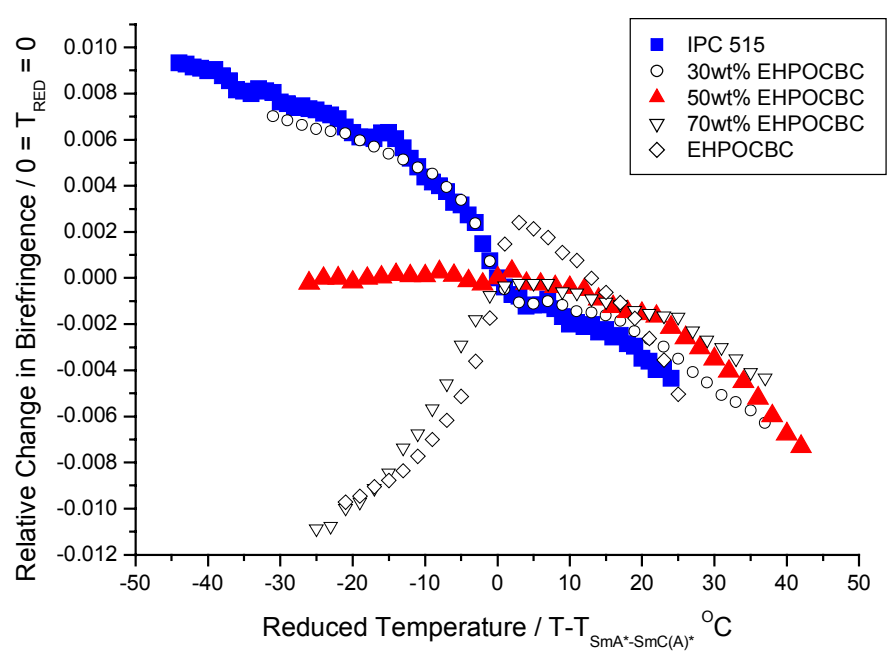

Figure 6. Relative change in birefringence on cooling for mixtures of IPC 515 and EHPOCBC, (for clarity the data corresponding to pure IPC 515,(red) and 50wt\% EHPOCBC,(blue), have been coloured).

The increase in birefringence of the $\mathrm{SmC}^{*}$-forming samples indicates that a more ordered structure is formed in the $\mathrm{SmC}^{*}$ phase, in the sense that there is a greater net alignment of mesogenic cores. One possible explanation is that the bulky isopinocampheol terminal group of IPC 515 promotes the type of molecule organization pictured in figure $2 \mathrm{a}$ in the $\mathrm{SmA}^{*}$ phase, giving this phase a strongly 
undulated structure. Such a packing arrangement is however not compatible with a $\mathrm{SmC}^{*}$ phase, where there is a non-zero macroscopically observable director tilt. Therefore, the transition to $\mathrm{SmC}^{*}$ may be accompanied by a rearrangement to the other space filling organization, that of locally antiparallel molecules. Such a molecule organization has been reported to be observed in both $\mathrm{SmA}^{*}$ and $\mathrm{SmC}^{*}$ phases [12, 13]. This would effectively lead to an increased parallelism of the molecular cores, which would fit well with the changes in optical properties. The strong first-order nature of the phase transition would be a natural result of this fairly drastic molecule reorganization.

One can analyze the situation also from the other extreme viewpoint, neglecting packing efficiency considerations and instead including the thermal motion in the analysis, in particular the very fast rotation around the molecular axis of inertia. The bent shape of IPC 515 gives the axis of inertia quite an angle to the multi-ring core part of the molecule. The rotation will will thus lead to an effective decrease in birefringence since the macroscopic optical properties will reflect all rotation angles, two of which corresponds to the core structure tilted some 10-15 degrees away from the optic axis, in opposite directions. This is certainly the situation in the $\mathrm{SmA}^{*}$ phase, but it is well known that the tilt in the SmC* phase biases the molecular rotation, so the optical properties could be expected to be affected from this. In this particular case, the bent shape of the molecule may lead to an enhancement of that effect. We know from the by now well-established analysis of achiral bent-core liquid crystals that such molecules tend to tilt with the bend plane perpendicular to the tilting direction. To some extent we can certainly expect that this will apply also to the IPC 515 molecule, i.e. the molecules will in the $\mathrm{SmC}^{*}$ phase spend considerably more time with the bend plane perpendicular to the tilting direction than in other rotated states. This will lead to a considerable increase in effective birefringence in a surface-stabilized planar sample, because not only is the rotation strongly biased, but the most likely state is also with the phenyl rings aligned along the light transmission direction. In other words, the orientation with the maximum anisotropy in polarizability is strongly favored. The two cases are illustrated in Figure 7. This could play an important role in explaining the distinct increase in birefringence observed below the $\mathrm{SmA}^{*}-\mathrm{SmC}^{*}$ transition.

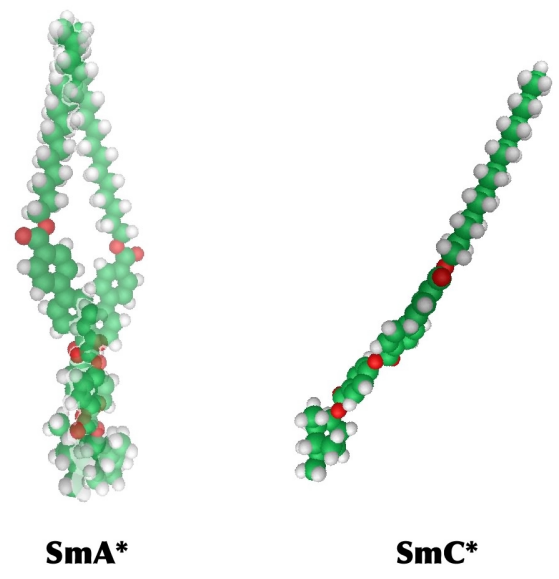

Figure 7. Possible situations in $\mathrm{SmA}^{*}$ and $\mathrm{SmC}^{*}$, giving the $\mathrm{SmC}^{*}$ phase considerably larger birefringence. For explanations, see text.

Plots of the relative birefringence as a function of temperature and applied field are shown in Figure 8 for IPC 515 and EHPOCBC. These data were taken for samples in $2 \mu \mathrm{m}$ cells, allowing the helix to be quenched. These plots clearly show that there is negligible field-dependence of the birefringence for IPC 515. This is true in both the 
$\mathrm{SmA}^{*}$ and the $\mathrm{SmC}^{*}$ phases. The absence of any field induced increase in birefringence is an indication that the electroclinic effect is most probably approximated by a fluctuation-mediated field induced tilt, as opposed to any biasing of tilted and uncorrelated molecules.
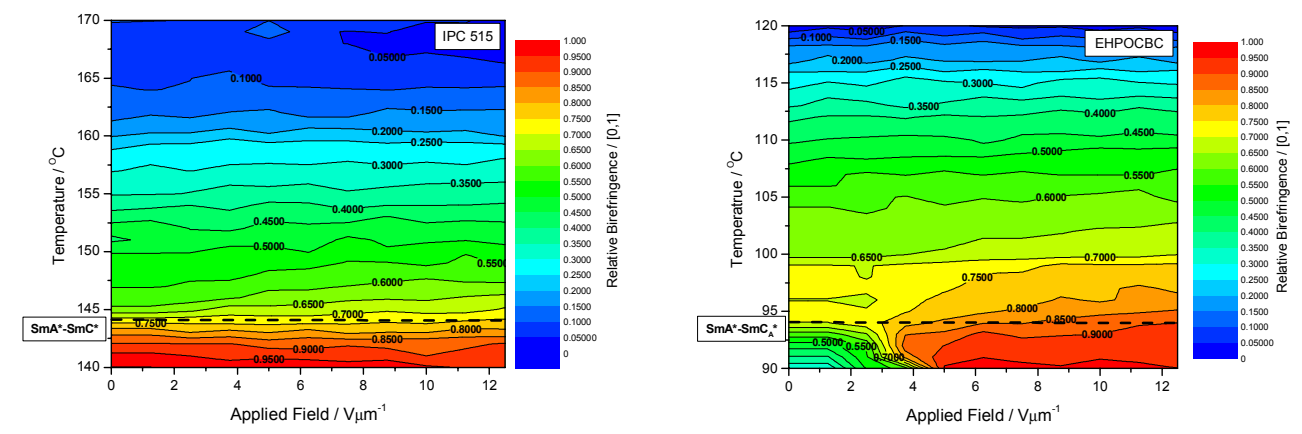

Figure 8. Contour plots of the relative birefringence versus temperature and applied field for the pure compounds IPC 515 and EHPOCBC.

In contrast, the $\mathrm{SmA}^{*}$ phase of $\mathrm{EHPOCBC}$ shows a definite increase in birefringence with applied field up to approximately $7^{\mathrm{O}} \mathrm{C}$ above the phase transition. This indicates that there is an ordering of the mesogenic cores effected by the applied field. The increase in birefringence with applied field can be attributed to the quenching of the fluctuation by the field. Measurement of the absolute birefringence of samples of IPC 515 and EHPOCBC indicate that the birefringence of the SmA* phase of EHPOCBC is greater than that of IPC 515, as would be expected for a nonundulating phase in comparison with a strongly undulating phase, but the difference can equally well be attributed to a larger inclination between the mesogenic core and the axis of inertia in IPC 515.

\section{CONCLUSIONS}

We have analysed the properties of a ferroelectric liquid crystal, the molecule of which contains a bulky rigid chiral end group, in relation to the possibility of exhibiting an asymmetric diffuse cone model $\mathrm{SmA}^{*}{ }_{-} \mathrm{SmC}^{*}$ transition. On the one hand, the layer shrinkage observed and the resulting formation of zig zag textural defects indicates that a transition of this type is not present. The formation of the $\mathrm{SmC}^{*}$ phase is connected to an increased average molecular tilt. On the other hand, the distinctly larger birefringence in $\mathrm{SmC}^{*}$ as compared to $\mathrm{SmA}^{*}$ cannot be explained by a simple 'non-de Vries-like' model. Clearly, the mesogenic cores must be more ordered in $\mathrm{SmC}^{*}$ than in $\mathrm{SmA}^{*}$ to account for this effect. Our results are thus slightly contradictory and we can at this stage draw no clear-cut conclusions regarding the type of $\mathrm{SmA}^{*}-\mathrm{SmC}^{*}$ transition promoted by using molecular structures with bulky end groups. We have however pointed out two possible approaches towards explaining the behavior, one assuming a different type of molecular packing in $\mathrm{SmA}^{*}$ and $\mathrm{SmC}_{\mathrm{A}} *$, the other involving the effect of the bent molecular shape on the rotational biasing in the $\mathrm{SmC}^{*}$ phase.

Finally, it is is interesting to note that the related AFLC compound EHPOCBC in the region directly above the $\mathrm{SmA}^{*}-\mathrm{SmC}_{\mathrm{A}}{ }^{*}$ transition exhibits properties that follow from the diffuse cone model, namely, large induced polarisation, significant birefringence change on applied field, and a large electroclinic effect. A possible 
explanation of the difference in transitional behaviour of IPC 515 and EHPOCBC is that the tilting transition in the latter case is mainly second-order, whereas the SmA*SmC* transition of IPC 515 is strongly first-order.

\section{ACKNOWLEDGEMENTS}

This work has been supported by the SAMPA European Network.

\section{REFERENCES}

1. J. P. F.Lagerwall, F. Giesselmann, M. D. Radcliffe, Phys. Rev. E, 66, 031703, (2002)

2. F. Giesselmann, J. P. F. Lagerwall, G. Andersson, M. D. Radcliffe, Phys. Rev. E, 66, 051704, (2000)

3. Y. Takanishi, Y. Ouchi, H. Takezoe, A. Fukada, A. Mochizuki, M. Nakatsuka, Jpn. J. Appl. Phys., 229, L984, (1990)

4. M. D. Radcliffe, M. L. Brostrom, K. Epstein, A. Rappaport, B. Thomas, R. Shao, N. A. Clark, Liq. Cryst., 26, 789, (1999)

5. M. S. Spector, P. A. Heiney, J. Naciri, B. T. Weslowski, D. B. Holt, R. Shashidar, Phys. Rev. E, 61, 1579, (2000)

6. F. Giesselmann, P.Zugenmaier, I. Dierking, S. T. Lagerwall, B. Stebler, M. Kaspar, V. Hamplova, M. Glogarova, Phys. Rev. E, 60, 598, (1999)

7. T. P. Rieker, N. A. Clark, G. S. Smith, D. S. Parmar, E. B. Sirota, C. R. Safinya, Phys. Rev. Lett., 59, 2658, (1987)

8. A. de Vries, A. Ekachai, N. Spielberg, Mol. Cryst. Liq. Cryst. Lett., 49, 143, (1979)

9. F. Giesselmann, A. Langhoff and P. Zugenmaier, Liq. Cryst., 23, 927 (1997)

10. K. Miyasato, S. Abe, H. Takezoe, A. Fukada, Jpn, J. Appl. Phys., 22, L661, (1983)

11. Physical Properties of Liquid Crystals:Nematics, Ed. D.Dumar, A.Fukada, G.Luckhurst, INSPEC, (2000), 315

12. N. H. Tinh, F. Hardouin, C. Destraade, J. Phys.(Paris), 43, 1127, (1982)

13. F. Hardouin, N. H. Tinh, M. F. Achard, A. Levelut, J. Phys. (Paris), Lett., 43, 327 , (1982)

14. M. Skarabot, K. Kocevar, R. Blinc, G. Heppke, I. Musevic, Phys. Rev. E, 59, R1323, (1999)

15. K. Ema, H. Yao, I. Kawamura, T. Chan, C. W. Garland, Phys. Rev. E, 47, 1203, (1993) 